\title{
BMJ Open Predictors of hospitalisations and emergency department presentations shortly after entering a residential aged care facility in Australia: a retrospective cohort study
}

\author{
Maria C Inacio (D) , ${ }^{1,2}$ Robert N Jorissen, ${ }^{2}$ Steve Wesselingh, ${ }^{3}$ Janet K Sluggett, ${ }^{1,2}$ \\ Craig Whitehead, ${ }^{4,5}$ John Maddison, ${ }^{6}$ John Forward, ${ }^{6}$ Alice Bourke, ${ }^{7}$ \\ Gillian Harvey, ${ }^{8}$ Maria Crotty (D) , ${ }^{4,5}$ STAAR-SA Study Collaborators
}

To cite: Inacio MC, Jorissen RN, Wesselingh S, et al. Predictors of hospitalisations and emergency department presentations shortly after entering a residential aged care facility in Australia: a retrospective cohort study. BMJ Open 2021;11:e057247. doi:10.1136/ bmjopen-2021-057247

- Prepublication history and additional supplemental material for this paper are available online. To view these files, please visit the journal online (http://dx.doi.org/10.1136/ bmjopen-2021-057247)

Received 10 September 2021 Accepted 20 0ctober 2021
Check for updates

(c) Author(s) (or their employer(s)) 2022. Re-use permitted under CC BY-NC. No commercial re-use. See rights and permissions. Published by BMJ.

For numbered affiliations see end of article.

Correspondence to

Dr Maria C Inacio;

Maria.inacio@sahmri.com

\section{ABSTRACT}

Objectives To: (1) examine the 90-day incidence of unplanned hospitalisation and emergency department (ED) presentations after residential aged care facility (RACF) entry, (2) examine individual-related, facility-related, medication-related, system-related and healthcare-related predictors of these outcomes and (3) create individual risk profiles.

Design Retrospective cohort study using the Registry

of Senior Australians. Fine-Gray models estimated subdistribution HRs and $95 \%$ Cls. Harrell's C-index assessed risk models' predictive ability.

Setting and participants Individuals aged $\geq 65$ years old entering a RACF as permanent residents in three Australian states between 1 January 2013 and 31 December 2016 ( $\mathrm{N}=116192$ individuals in $1967 \mathrm{RACFs}$ ).

Predictors examined Individual-related, facility-related, medication-related, system and healthcare-related predictors ascertained at assessments or within 90 days, 6 months or 1 year prior to RACF entry.

Outcome measures 90-day unplanned hospitalisation and ED presentation post-RACF entry.

Results The cohort median age was 85 years old (IQR $80-89), 62 \%(\mathrm{~N}=71861)$ were women, and $50.5 \%$ $(\mathrm{N}=58714)$ had dementia. The 90-day incidence of unplanned hospitalisations was $18.0 \%$ ( $\mathrm{N}=20919)$ and $22.6 \%(N=26242)$ had ED presentations. There were 34 predictors of unplanned hospitalisations and 34 predictors of ED presentations identified, 27 common to both outcomes and 7 were unique to each. The hospitalisation and ED presentation models out-of-sample Harrell's C-index was 0.664 (95\% $\mathrm{Cl} 0.657$ to 0.672 ) and 0.655 (95\% Cl 0.648 to 0.662), respectively. Some common predictors of high risk of unplanned hospitalisation and ED presentations included: being a man, age, delirium history, higher activity of daily living, behavioural and complex care needs, as well as history, number and recency of healthcare use (including hospital, general practitioners attendances), experience of a high sedative load and several medications.

Conclusions Within 90 days of RACF entry, $18.0 \%$ of individuals had unplanned hospitalisations and $22.6 \%$ had

\section{Strengths and limitations of this study}

- Our study provides population-based estimates of the incidence and predictive factors for unplanned hospitalisations and emergency department (ED) presentations for aged care recipients of three Australian states (covering 68\% of Australians residential aged care facility residents) and are generalisable to the Australian population and Western countries with similar aged care sectors and similarly ageing populations.

- Our models can moderately well categorise individuals according to their risk of unplanned hospitalisations and ED presentation and provide insightful information about the factors that contribute to these risks, which can be used by clinicians and aged care providers in their care planning and risk mitigation strategies.

- Only public hospitalisation data were used to ascertain outcomes in this study.

- Our analysis focused on predictive models for the studied outcomes that provide interpretable estimates for the factors studied, which likely resulted in lower performing models than more sophisticated machine learning models.

- We cannot infer causality from any of the relationships presented in our findings given the nature of the data and analysis.

ED presentations. Several predictors, including modifiable factors, were identified at the time of care entry. This is an actionable period for targeting individuals at risk of hospitalisations.

\section{INTRODUCTION}

In 2016, in 26 Organisation for Economic Co-operations and Development participant countries, almost five million older people lived in residential aged care facilities (RACF) (ie, nursing homes or long-term care 
facilities). ${ }^{1}$ In Australia, where approximately 240000 people live as permanent residents of RACFs each year, who have a $25 \%-30 \%$ reported yearly mortality rate, an approximately 70000 new residents enter RACFs each year. ${ }^{23}$ Aged care services in Australia, which include longterm care in RACFs or at home and other transition, respite or home support services, are subsidised by the federal government, while the healthcare services Australians receive are funded by both state (hospitals) and federal government (eg, health services, procedures) ${ }^{34}$ Over the last decade individuals entering RACFs have increasingly entered care older, with a higher burden of functional limitations, greater burden of comorbid conditions, and significantly frailer. ${ }^{5}$ It is well documented that the period of transition into permanent care is one of significant vulnerability for older individuals. The events leading to entry into an RACF (eg, major changes in health status) combined with the transition itself, which includes unfamiliarity with a new environment, new carers, changes in healthcare providers, medications and routines, can pose challenges to individuals, leading to poor health events after entering care. ${ }^{6-12}$

Older individuals, especially those living in RACFs have frequent hospitalisations. ${ }^{13} 14$ In Australia, 37\% of RACF residents had at least one hospitalisation and $37 \%$ at least one emergency department (ED) presentation in 2018/2019. ${ }^{14}$ The period of entry into an RACF is also one of significant risk for residents to experience events that may lead to hospitalisations, ${ }^{8} 15$ with international estimates of 'short-term' hospitalisations after entering care ranging from $6.8 \%$ to $62 \% .{ }^{8915}$ To date, in Australia, we have population-based estimates of hospitalisation of individuals in RACFs ${ }^{14}$ but no estimates of unplanned hospitalisations or ED encounters, important measures of potential adverse events occurrences at care transitions. Frequent care transitions can be distressing, particularly for the $50 \%$ of residents who have been diagnosed with dementia entering RACFs, ${ }^{5}$ and can be hampered by quality and safety issues such as problems accessing urgent health and medication information, lack of person-centred care and poor communication and handover. ${ }^{16}$ As entry into permanent residential care is a period where significant time is spent on assessing individuals' care needs, creating and implementing care plans, and engaging with allied health providers, risk profiling of individuals during this important period can inform care plans and risk mitigation strategies.

Using the national and state-based integrated healthcare and aged care data from the Registry of Senior Australians (ROSA) historical national cohort ${ }^{5}$ we have: (1) identified the incidence of unplanned hospitalisations and ED presentations within 90 days in individuals entering permanent care; (2) examined individual, medication, system and healthcare factors, known at RACF entry, and their associations of these events and (3) developed risk profiles for these events.

\section{METHODS}

\section{Study design, setting and data source}

A retrospective cohort study was conducted using the ROSA. ${ }^{5}$ Briefly, ROSA contains deidentified linked information from the Australian Institute of Health and Welfare's National Aged Care Data Clearinghouse, which includes the National Death Index, and the Australian Government Medicare Benefits Schedule (MBS) and Pharmaceutical Benefits Scheme datasets, and state health authorities' ED and inpatient hospitalisations data collections. ROSA captures individuals being assessed for eligibility and accessing aged care services for which an eligibility assessment is required, namely residential aged care, home care packages, transition care and respite care.

\section{Study cohort}

The cohort includes all non-indigenous individuals $\geq 65$ years old who had a first-time entry as a permanent resident in an RACF in the states of South Australia (SA), New South Wales (NSW) and Victoria (VIC), between 1 January 2013 and 31 December 2016 and who did not receive Department of Veterans' Affairs subsidised services and were not considered residents in palliative care at entry ( $N=120221)$. Aged care recipients from these three states represent $68 \%$ of the national cohort. Individuals missing the basic assessment to determine fundamental care needs, which is required for government funding allocation (named the 'Aged Care Funding Instrument') were excluded $(\mathrm{N}=4029,3 \%)$. The final study cohort was $\mathrm{N}=116192$.

\section{Outcomes of interest}

Unplanned hospitalisation and ED presentations at public hospitals within 90 days (or 'short term') of a first-time entry as a permanent RACF resident were the outcomes of interest. Unplanned hospitalisations were hospitalisations where the 'Admission Urgency Status' specified 'Emergency' and not 'Scheduled.' The follow-up period was 1 January 2013-31 March 2017.

\section{Predictors of interest}

The candidate variables for our prediction models included individuals' characteristics, their RACFs' characteristics, pharmaceutical claims history and health services utilisation, inclusive of primary care, hospitals and specialist's healthcare services, which were available in the ROSA datasets.

Individual factors (table 1 and online supplemental table 1) ascertained from assessments performed for service eligibility determination or entry into permanent care included: date of birth, sex, partner status, frailty index score, ${ }^{17}$ levels of need regarding activities of daily living (ADL), levels of need regarding cognition and behaviour, levels of need regarding complex healthcare, health conditions and Socio-Economic Indexes for Areas', relative socioeconomic disadvantage index and education and occupation index. ${ }^{18}$ Geriatric health 
Table 1 Study cohort description, highlights of individual, medication and facility-related factors by unplanned hospital admission or emergency department presentation status within 90 days of entry into a residential aged care facility

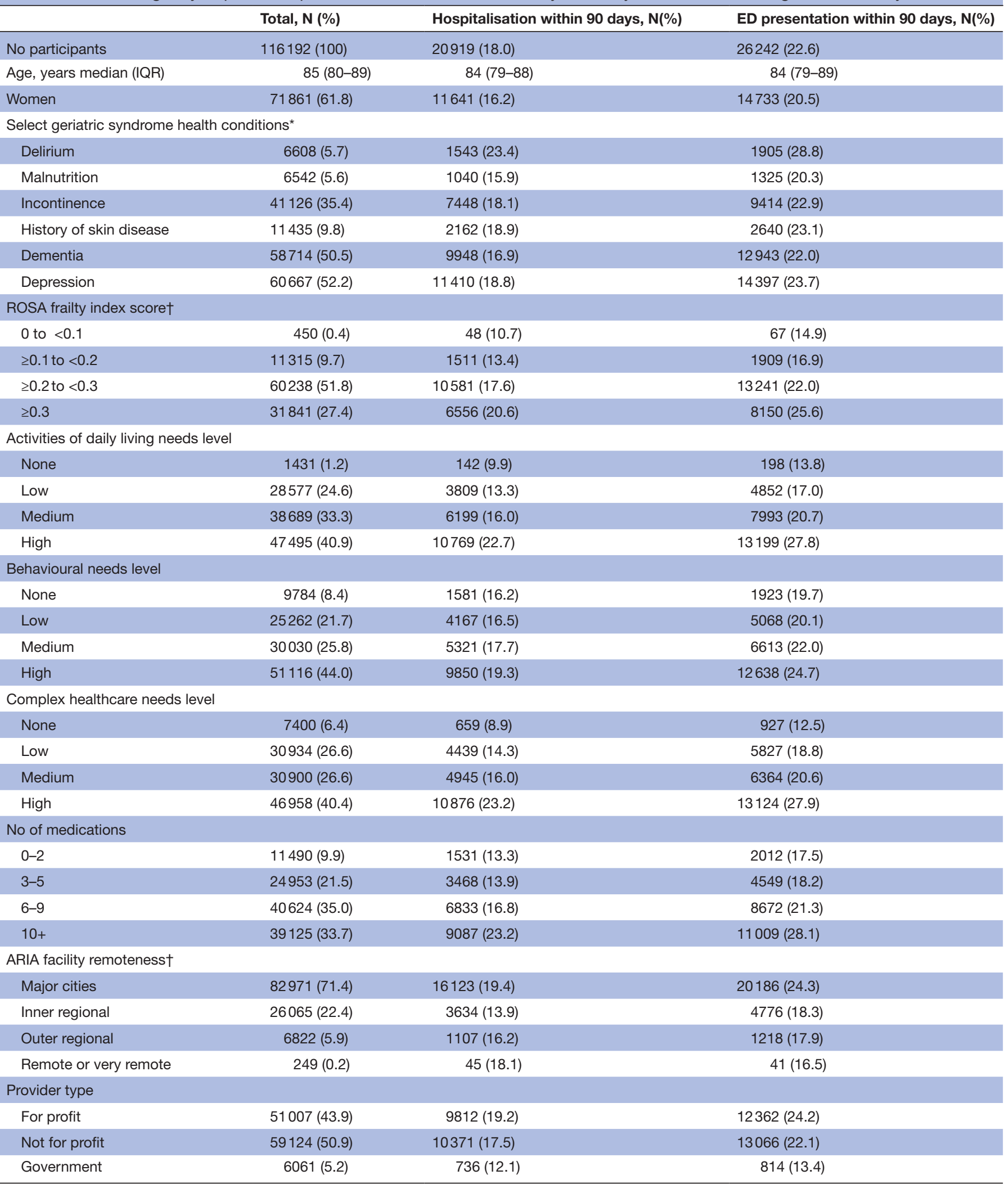

*All, except dementia, were ascertained from the aged care eligibility and entry into care assessments. Dementia was ascertained from the aged care eligibility assessment, entry into care assessment and the RxRisk-V medication-based comorbidity condition indicator for dementia.

tMissing data: ROSA Frailty Index Score $n=12348(10.6 \%)$, ARIA remoteness $n=85(0.07 \%)$

ARIA, Accessibility/Remoteness Index of Australia; ED, emergency department; IQR, Interquartile range; ROSA, Registry of Senior Australians. 
conditions were ascertained from the union of data from both assessments and when appropriate (eg, for dementia) both assessments and the condition from the prescription-based comorbidity measure RxRisk-V in the 6 months prior to cohort entry. The RxRisk-V also ascertained the count of individuals' co-morbid conditions using the 6 months prior to cohort entry history. For falls ascertainment specifically, in addition to the assessments, a 1-year history of public hospitalisations where fall was reported (using ICD-10-AM codes W00*, W01*, W03*W19* and R29.6) was also used.

The facility characteristics examined included: location of facility (state, geographical remoteness) ${ }^{19}$ and type (not for profit, for profit or government).

Medication-related factors (table 1 and online supplemental table 2) were ascertained from pharmaceutical claims records in the 90-day period prior to RACF entry and included: number of medications supplied (categorised by Anatomical, Therapeutic and Chemical classification (ATC) codes, fifth level chemical substance), ${ }^{20}$ sedative load rating (ie, cumulative effect of medications with sedative properties) ${ }^{21}$ and medication class (ie, ATC fourth level, chemical, pharmacological or therapeutic subgroup level).

Healthcare-related factors (table 2 and online supplemental table 3) ascertained using the history of public hospitalisations in the year prior to RACF entry included: number of hospitalisations (unplanned and potentially preventable hospitalisations), ${ }^{21}$ number of ED presentations (overall and potentially preventable), cumulative length of hospital stays and 30-day history of hospitalisations or ED presentations (unplanned and potentially preventable hospitalisations). Additional factors were ascertained using the MBS subsidised services (online supplemental table 4) in the year prior to care entry and included: primary healthcare (eg, general practice (GP) attendances, health assessments) or specialist services frequently used by older individuals (eg, geriatric services).

\section{Statistical analysis}

The cohort was described using means, SD, medians, $\mathrm{IQR}$, frequencies and proportions. Fine and Gray models with death as a competing risk, using a modified version of the Hosmer and Lemeshow's purposeful selection of variables approach, for each outcome were employed. ${ }^{22}$ This approach involved the repeated elimination of candidate covariates whose omission were not statistically significant from application of the likelihood ratio test; these omissions were stopped when removal of covariates were found to be statistically significant (using a $p=0.001$ cut-off due to large cohort size). Covariates with less than $5 \%$ prevalence (ie, some medications and health services) or collinear with other covariates (determined from Pearson correlation $>0.75$ ) were a priori omitted from model building. When collinearity was confirmed, covariates with fewer missing data or easier to model (ie, functional form) were chosen. Terms for covariate effect modification (interaction) with either age or sex were added if they were considered statistically significant from application of the likelihood ratio test, and then simplified post hoc as appropriate. Continuous variables' functional forms were examined, and best distributions modelled, which included non-linear terms or truncation for certain variables. Subdistribution sHRs and 95\% CIs were presented. Models' discrimination was examined by calculating Harrell's C-index, which assesses the proportion of subject pairs that have the same ordering of predicted and observed survival times, within-sample using a 10 -fold cross-validation and out-of-sample using a cohort of individuals not included in the derivation cohort, that is, $\mathrm{N}=26314$ city-dwelling individuals with aged care eligibility assessments in 2012. All calculations used complete-case analysis and facility remoteness was the variable with greatest number of missing records $(0.07 \%$ missing $)$.

\section{Patient and public involvement}

Patients or the public were not involved in the design, or conduct, or reporting of our research. Consumer representatives will be consulted in the dissemination of this work.

\section{RESULTS \\ Cohort description}

In the 116192 individuals studied the median age was 85 years (IQR 80-89), 61.8\% ( $\mathrm{N}=71861)$ were women, $50.5 \%(\mathrm{~N}=58714)$ had dementia and $27.4 \%$ $(\mathrm{N}=31841)$ had a frailty index score of $\geq 0.3$ (most frail). See table 1 and online supplemental table 1 for the full cohort description. These individuals entered 1967 distinct RACFs, with 43.9\% ( $\mathrm{N}=51007)$ entering a for profit, $50.9 \%(\mathrm{~N}=59124)$ a non-profit and $5.2 \%$ $(\mathrm{N}=6061)$ a government managed facility. Of the cohort, $69.0 \%(\mathrm{~N}=80139)$ had at least one unplanned hospitalisation, $17.3 \%(\mathrm{~N}=20129)$ had at least one potentially preventable hospitalisation and $72.3 \%$ $(\mathrm{N}=83896)$ at least one ED presentation in the year prior to care entry (See table 2 and online supplemental tables 3 and 4 for hospital and other health services utilisation).

\section{Incidence of 90-day unplanned hospitalisations and ED presentations after care entry}

Within 90 days of RACF entry the crude incidence of individuals with unplanned hospitalisations was $18.0 \%(\mathrm{~N}=20919)$ and of ED presentations was $22.6 \%$ $(\mathrm{N}=26242)$. During the 90 days after entry into RACF 9.4\% ( $\mathrm{N}=10910)$ of individuals died (see online supplemental figure $\mathrm{S} 1$ for cumulative incidence curves).

The three most common groups of diagnosis leading to unplanned hospitalisations included: $22.1 \%$ for injury, poisoning and certain other consequences of external 
Table 2 Study cohort description, highlights of hospital and healthcare-related factors ${ }^{*}$ by unplanned hospital admission or emergency department presentation status within 90 days of entry into a residential aged care facility

\begin{tabular}{|c|c|c|c|}
\hline & Total, N (\%) & Hospitalisation within 90 days, $\mathrm{N}(\%)$ & ED presentation within 90 days, $\mathrm{N}(\%)$ \\
\hline No participants & $116192(100)$ & 20919 (18.0) & $26242(22.6)$ \\
\hline \multicolumn{4}{|c|}{ No unplanned hospitalisations } \\
\hline 1 & $41383(35.6)$ & $7097(17.1)$ & $8982(21.7)$ \\
\hline $2-4$ & $34321(29.5)$ & $8215(23.9)$ & 10035 (29.2) \\
\hline 0 & $96063(82.7)$ & $15477(16.1)$ & $19815(20.6)$ \\
\hline 1 & $14691(12.6)$ & 3505 (23.9) & $4236(28.8)$ \\
\hline $2-4$ & $4969(4.3)$ & $1712(34.5)$ & $1943(39.1)$ \\
\hline $5+$ & $469(0.4)$ & $225(48.0)$ & $248(52.9)$ \\
\hline $2-4$ & $40278(34.7)$ & $8921(22.1)$ & $11225(27.9)$ \\
\hline $5+$ & $9143(7.9)$ & $3003(32.8)$ & $3733(40.8)$ \\
\hline \multicolumn{4}{|c|}{ GP attendances (MBS Group A01) } \\
\hline $0-4$ & $23827(20.5)$ & $3535(14.8)$ & $4548(19.1)$ \\
\hline $5-10$ & $38948(33.5)$ & $6594(16.9)$ & $8387(21.5)$ \\
\hline $11+$ & $53417(46.0)$ & $10790(20.2)$ & 13307 (24.9) \\
\hline \multicolumn{4}{|c|}{ Services for patients in RAC facilities (MBS Group A35) } \\
\hline $0-4$ & $97056(83.5)$ & $17179(17.7)$ & $21658(22.3)$ \\
\hline $5-10$ & $15197(13.1)$ & $2885(19.0)$ & $3560(23.4)$ \\
\hline \multicolumn{4}{|c|}{ GP after hours attendances (MBS Group A22) } \\
\hline 0 & $77219(66.5)$ & $12994(16.8)$ & $16351(21.2)$ \\
\hline 1 & $20459(17.6)$ & $3911(19.1)$ & $4878(23.8)$ \\
\hline $2-4$ & $13562(11.7)$ & $2850(21.0)$ & $3535(26.1)$ \\
\hline $5+$ & $4952(4.3)$ & $1164(23.5)$ & $1478(29.8)$ \\
\hline
\end{tabular}

* All hospital and healthcare-related factors utilisation shown were in the year prior to residential aged care facility entry. GP, general practitioners; MBS, Medicare Benefits Schedule; RAC, residential aged care.

causes (International Statistical Classification of Diseases, 10th Revision, Australian Modification (ICD-10-AM) S00T98 codes), of which $7.8 \%$ were for fractures of subcapital section of femur (ICD-10-AM S72.03) and 6.7\% for fracture of intertrochanteric session of the femur (ICD-10-AM S72.11); $15.8 \%$ were for diseases of the respiratory system (ICD-10-AM J00-J99), of which 33.6\% were due to pneumonia unspecified (ICD-10-AM J18.9), and $18.7 \%$ due to pneumonitis due to food and vomit (ICD-10-AM J69.0); and $13.4 \%$ for diseases of the circulatory system (ICD10-AM I00-I99), of which $32.9 \%$ were due to congestive heart failure (ICD-10-AM I50.0) and 7.9\% for acute subendocardial myocardial infarction (ICD-10-AM I21.4). The three most common groups of diagnosis leading to ED presentations included: $27.9 \%$ for symptoms, signs and abnormal clinical findings (ICD-10-AM R00-R99), of which $23.5 \%$ was for tendency to fall (ICD-10-AM R29.6), 9.5\% for chest pain, unspecified (ICD-10-AM R07.4), and $8.3 \%$ for syncope and collapse (ICD-10-AM R55); $18.8 \%$ for injury, poisoning and certain other consequences of external causes (ICD-10-AM S00-T98), of which 9.6\% were for fracture of neck of femur (ICD-10-AM S72.00) 
A
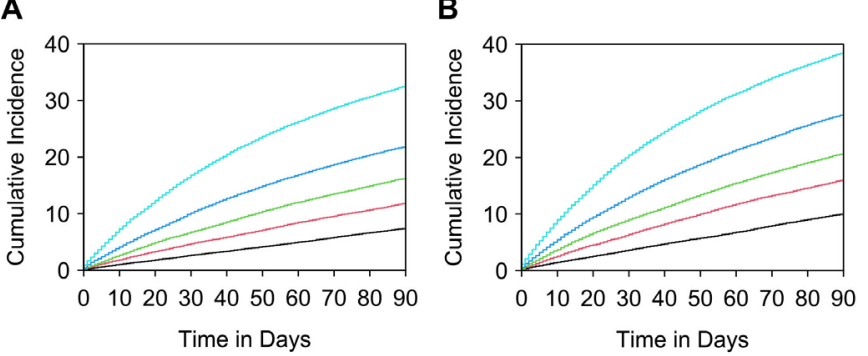

C

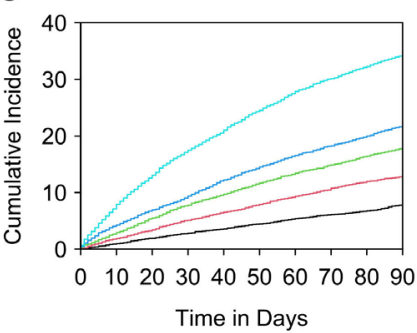

D

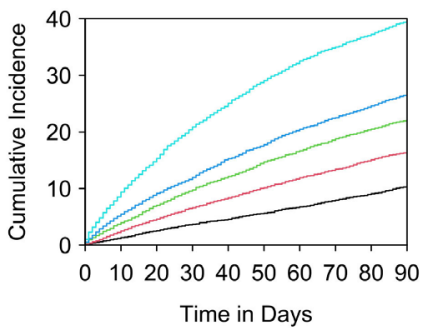

\begin{tabular}{lcc}
\hline & \multicolumn{2}{c}{ Harrell's C-index } \\
\cline { 2 - 3 } & $\begin{array}{c}\text { Hospitalisation } \\
\text { within 90 days }\end{array}$ & $\begin{array}{c}\text { ED presentation } \\
\text { within 90 days }\end{array}$ \\
\hline $\begin{array}{l}\text { In-sample 10-fold cross-validation } \\
\text { Using quintile groups }\end{array}$ & $0.658(95 \% \mathrm{Cl} 0.654-0.661)$ & $0.648(95 \% \mathrm{Cl} 0.644-0.651)$ \\
Using prediction scores & $0.667(95 \% \mathrm{Cl} 0.663-0.671)$ & $0.657(95 \% \mathrm{Cl} 0.653-0.660)$ \\
Out-of-sample validation & & \\
Using quintile groups & $0.655(95 \% \mathrm{Cl} 0.648-0.662)$ & $0.647(95 \% \mathrm{Cl} 0.641-0.654)$ \\
Using prediction scores & $0.664(95 \% \mathrm{Cl} 0.657-0.672)$ & $0.655(95 \% \mathrm{Cl} 0.648-0.662)$ \\
\hline
\end{tabular}

Figure 1 Cumulative incidence plots (after entry into a residential aged care facility) using quintiles of risk predictions for: (A) unplanned hospitalisation from insample 10-fold cross-validation; (B) emergency department presentation from in-sample 10-fold cross-validation; (C) unplanned hospitalisation from out-of-sample validation and (D) emergency department presentation from out-of-sample validation. The groups of quintiles of predicted risk, from lowest to highest, are coloured: black (lowest, first quintile)), red (second quintile), green (third quintile), blue (fourth quintile) and light blue (highest, fifth quintile).

$\mathrm{ED}=$ Emergency department.

and $7.1 \%$ for fracture of other parts of neck of femur (ICD-10-AM S72.08); and 11.9\% for diseases of the respiratory system (ICD-10-AM J00-J99), of which 18.7\% were for pneumonia unspecified (ICD-10-AM J18.9), and $18.0 \%$ for unspecified acute lower respiratory infection (ICD-10-AM J22). See online supplemental table 5 for full reasons for first hospitalisations and ED presentations for the cohort.

\section{Predictors of 90-day unplanned hospitalisation and ED presentations after care entry}

There were 34 predictors of unplanned hospitalisations and 34 predictors of ED presentations identified. Of the predictors identified, 27 were common to both outcomes and 7 were unique to each. The model estimating risk of unplanned hospitalisation and ED presentation outof-sample Harrell's C-index was 0.664 (95\% CI 0.657 to 0.672 ) and 0.655 (95\% CI 0.648 to 0.662 ), respectively (figure 1; online supplemental figure $\mathrm{S} 2$ for the receiver operator characteristic curve).
Common predictors associated with a higher risk of unplanned hospitalisation and ED presentation included (see table 3 for risk estimates and functional forms): being a man, being younger (after the age of 90), having history of delirium, higher ADL needs, higher behavioural needs, higher complex care needs, having entered the facility in 2013 compared with 2015, history, number and recency of hospitalisations and ED presentations in the year prior to entry, number of regularGP and GP urgent after hours attendances in the year prior, exposure to a high sedative load from medications dispensed in the 6 months prior to care entry, and the use of proton pump inhibitors (PPIs), sulfonamides diuretics (ie, furosemide, bumetanide), beta blockers, vitamin $\mathrm{K}$ antagonists (warfarin), organic nitrates, inhaled adrenergics in combination with corticosteroids or other drugs (ie, short-acting or long-acting beta2 agonist and corticosteroid combination products) and inhaled anticholinergics. Common factors associated with a lower risk of unplanned hospitalisations and ED presentations included: history of incontinence, no complex care needs compared with lower care needs, being from the state of VIC compared with NSW, entering a government funded facility compared with a non-profit, and anticholinesterases use in the 6 months prior to care entry.

Predictors of higher risk of unplanned hospitalisation only included: facility being in a major city or outer regional compared with inner regional areas, facility entry year 2016, use of systemic glucocorticoids and preparations inhibiting uric acid production (ie, allopurinol, febuxostat) in the 6 months prior to entry into RACF. Factors associated with a lower risk of unplanned hospitalisation only included: living with dementia and facility in SA compared with NSW.

Predictors of a higher risk of ED presentation only included: facility located in a major city compared with inner regional, number of medications individuals had dispensed in the last 6 months, and number of urgent after-hours attendances in the year prior to care entry. Factors associated with a lower risk of ED presentation only included: facility not in a major city in SA compared with inner city NSW, use of HMG CoA reductase inhibitors (statins) and use of two or more health services in RACFs in the year prior to permanent care entry.

\section{DISCUSSION}

In this large cohort study of new residents of 1967 RACFs between 2013 and 2017, we determined that $18.0 \%$ of individuals had an unplanned hospitalisation and $22.6 \%$ had an ED presentation within 90 days of entering care. We also identified 27 factors, including potentially modifiable ones such as exposure to high medication sedative load, that are common predictors of both unplanned hospitalisations and ED presentation and an additional 7 that are unique to each of the outcomes examined. We have demonstrated that moderately well performing risk predictions tools for whether an individual will get 
Table 3 Predictors of unplanned hospitalisation and emergency department presentations within 90 days of entry into a residential aged care facility

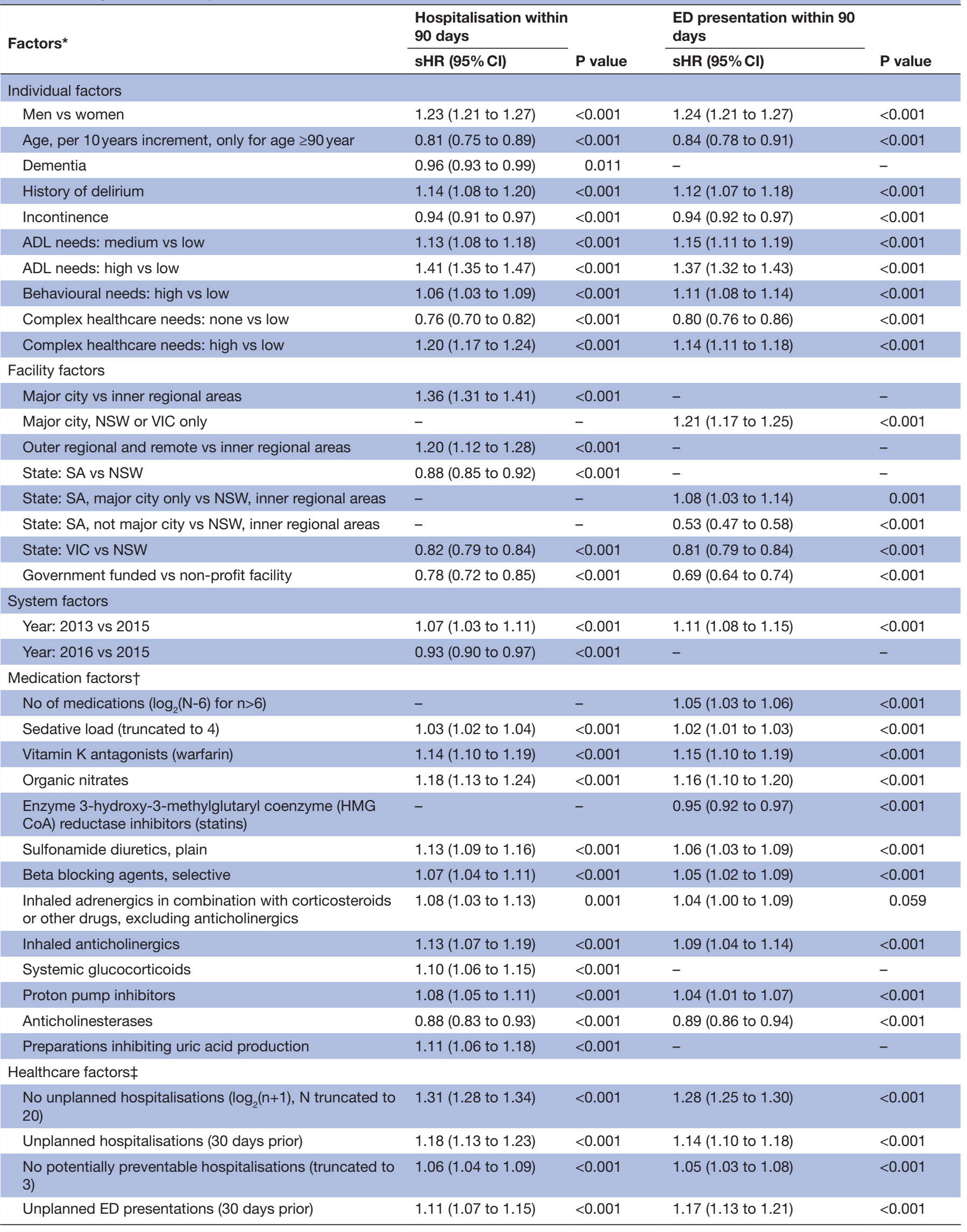


Table 3 Continued

\begin{tabular}{|c|c|c|c|c|}
\hline \multirow{2}{*}{ Factors* } & \multirow{2}{*}{$\begin{array}{l}\text { Hospitalisation within } \\
90 \text { days } \\
\text { sHR }(95 \% \mathrm{Cl})\end{array}$} & \multirow[b]{2}{*}{$P$ value } & \multirow{2}{*}{$\begin{array}{l}\text { ED presentation within } 90 \\
\text { days } \\
\text { sHR }(95 \% \mathrm{Cl})\end{array}$} & \multirow[b]{2}{*}{$P$ value } \\
\hline & & & & \\
\hline Two or more services for patients in RACFs & - & - & 0.94 (0.92 to 0.97$)$ & $<0.001$ \\
\hline No urgent after-hours attendances (truncated to 3 ) & - & - & $1.03(1.02$ to 1.05$)$ & $<0.001$ \\
\hline
\end{tabular}

*If functional form not specified in parenthesis following factor, factor was binary (yes vs no) or linear (per unit increment). †See online supplemental table 3 for specific medications' Anatomical, Therapeutic and Chemical classification codes. fUnless specified look back period for variable ascertainment is for the year prior to entry into permanent RACF entry. $\mathrm{ADL}$, activities of daily living; $\mathrm{Cl}$, Confidence interval; ED, emergency department; GP, general practitioners; NSW, New South Wales; RACF, residential aged care facility; SA, South Australia; sHR, subdistribution hazard ratio; VIC, Victoria.

hospitalised or have an ED presentation can be derived from integrated aged care and healthcare information from the period around entry into RACF. Given the critical period that the transition time into RACF is for older individuals, this type of risk profiling for these common events can be valuable for aged care and healthcare providers to inform and implement individually tailored risk mitigation strategies.

Our analysis confirms that men, and individuals with a history of delirium, higher levels of ADL limitations, behavioural needs and complex healthcare conditions, which are known risk factors for RACFs' residents hospitalisations, ${ }^{6} 1523-25$ are also associated with unplanned hospitalisations shortly after entry into RACF in a national cohort of new aged care residents. Similar to other studies we have found the relationship of age and hospitalisations and ED presentation to be complex and in our case we found that only after an individual was 90 years old was an inverse association between age and risk of these events obvious. ${ }^{6}{ }^{15}$ We also determined that incontinence was associated with a lower risk of hospitalisation, which has been reported by O'Malley et al in a large study of 687956 US nursing home residents. ${ }^{24}$

Given the high prevalence of polypharmacy among individuals in RACFs, it is not unexpected that several medication classes, including the summative indicator of sedative load, were found to be associated with hospitalisations and ED presentations. As previously described, potentially high sedative burden, puts individuals at a higher risk of certain hospitalisations, most commonly for falls. ${ }^{26}$ The count of medications alone was also found to be a predictor of ED presentations, but not unplanned hospitalisations. Polypharmacy, a newly implemented national quality indicator in Australian RACFs, ${ }^{28}$ has been associated with hospitalisations in a study of RACF residents ${ }^{29}$ and is a flag for a need to examine appropriateness of medication use. Some of the associations identified likely reflect the most common reasons for hospitalisations and ED presentations, or individuals with more severe health conditions. For example, several cardiovascular (sulfonamide diuretics, beta blockers, warfarin, nitrates) and respiratory medications (inhaled short-acting or long-acting beta2 agonist and corticosteroid combination products, inhaled anticholinergics and systemic corticosteroids) were associated with unplanned hospitalisations and/or ED presentations and diseases of the respiratory and circulatory systems were among the most common reasons for these unplanned events. Individuals taking warfarin who experience a fall or injury may also be transferred to hospital due to concern about bleeding risk. ${ }^{30}$ Individuals prescribed these medication classes could benefit from a medication review on entry to the RACF. Closer review of asthma and chronic obstructive pulmonary disease action plans, review of inhaler technique, staff training and flags for vaccinations in electronic medication management systems were recommended in a previous root cause analysis of infectionrelated hospitalisations from South Australian RACFs. ${ }^{31}$ PPIs, which were dispensed to $45 \%$ of the cohort prior to RACF entry, were associated with both studied outcomes. PPI use has been associated with pneumonia, fractures and Clostridioides difficile infection. ${ }^{32}$ There may be opportunities to deprescribe PPIs among residents who are treated for $>8$ weeks or 'step-down' treatment among high-dose users. ${ }^{33}$ Further, we found anticholinesterases to be associated with a lower risk of both hospitalisation and ED presentations. In Australia, anticholinesterases are subsidised for managing cognitive symptoms of mildto-moderate symptoms of Alzheimer's disease and are associated with adverse events such as sedation, dizziness and syncope. ${ }^{34}$ Dementia was also found to be associated with a lower risk of hospitalisation, which was also identified by a large US study and reported to be associated with a lower risk of hospitalisation before death in a recent systematic review. ${ }^{24} 35$ However, individuals with greater behavioural needs were more likely to be hospitalised which suggests dementia severity may impact hospitalisation decisions as well as recognition of changes to an individual's health status.

Both type of RACF and its geographical location were predictors of unplanned hospitalisations and ED presentations. Payer models for RACF are often reported to 
be associated with quality of care provided and events such as hospitalisations. ${ }^{36}{ }^{37}$ However, this relationship is dependent on country-specific models for aged care delivery. ${ }^{36}{ }^{37}$ In line with Australian Royal Commission into Aged Care Quality and Safety reports, ${ }^{38}$ which looked at more than 40 indicators of quality and safety of care, several of which were hospitalisations for specific health events, we found government run facilities were consistently associated with lower incidence of unplanned hospitalisations and ED presentations. Canada, which like Australia, has a largely government subsidised aged care sector with resident contributions, delivered in facilities that are mostly non-profit, followed by for profit and then government managed, has also found similar results regarding hospitalisations. ${ }^{37}$ Differences in regionality were also reported by the Royal Commission, but with less consistency than for types of facility. ${ }^{38}$ Also, statebased differences were observed, and are likely reflective of varying state-based policies, access to pathology or medical imaging at the RACF, ${ }^{39}$ differences in private hospitals use for unplanned hospitalisations, ${ }^{13}$ and differences in hospital avoidance strategies (eg, extended care paramedics, hospital avoidance programmes),${ }^{40} 41$ which are not captured in this analysis. Another factor we determined to contribute to the risk of unplanned hospitalisation and ED presentation was year of RACF entry, with the most recent years being less likely to be associated with hospitalisations. While it is possible that less hospitalisations are truly occurring in more recent years because of the numerous efforts to reduce hospitalisations in these high-risk individuals, it is also possible individuals may be hospitalised more often in private hospitals, which would therefore not be captured in our analysis and is an important consideration in the interpretation of these findings.

The strongest predictors of hospitalisation and ED presentation we identified included the history of hospitalisation in the 30 days prior as well as the number of hospitalisations in the year prior to RACF entry, which has also been reported by the international literature for both people living in RACFs and in the community. ${ }^{243642}{ }^{43}$ We also identified other health service factors that contribute to the prediction of hospitalisations, including the number of regular and urgent after hour GP attendances in the year prior to RACF entry, which we have also reported in a cohort of older Australians at the point of their aged care eligibility assessment. ${ }^{44}$ Similarly, other studies have suggested that frequency of primary care encounters alone can be associated with a higher incidence of hospitalisations and ED presentations, and that continuity and quality of primary care relationships could be potential drivers for reducing the occurrence of these events. ${ }^{12}$ Finally, we identified access to in-residential aged care primary care attendances, evidenced by subsidised services for residential aged care settings only, to be associated with a $6 \%$ lower risk of ED presentations in those entering RACFs. We investigated how these individuals had claims for these events in the year prior to RACF entry and determined that they accessed residential respite care at RACF in the year prior, which a large proportion (about 53\% of the current cohort) of individuals in Australia usually do before entering care permanently.

The risk profiling models presented have moderate predictive performance (Harrell $\mathrm{C}$ between 0.65 and 0.66 ), which is similar to risk profiling models we have created for these outcomes in individuals living in the community and recently assessed for aged care services eligibility. ${ }^{44}$ Our estimates are also within the range of published predictive models of hospitalisations and ED presentations in older individuals in general, which have had predictive abilities from poor (0.53) to good (0.83) with few models achieving good predictive ability (ie, c $>0.8) .{ }^{42}{ }^{43}$ Additionally, a recent study of an Australian risk profiling tool for RACF residents was published proposing risk factors for hospitalisations but did not provide its model predictive ability. ${ }^{25}$

Our study has several limitations. As $92 \%$ of emergency hospitalisations are reportedly captured in public hospitals in Australia, we expect an underestimation of the unplanned hospitalisation and ED encounters in our analysis, but we do not have any reasons to believe this would be differential by the factors analysed. ${ }^{13}$ Our analysis focused on predictive models for the studied outcomes that provided interpretable estimates for the factors included, which likely resulted in lower performing models than more sophisticated machine learning models. We cannot infer causality from any of the relationships presented in our findings given the nature of the data and analysis performed and will consider our hypothesis generating findings in future analysis that support causal inference examination. Our integration of health and aged care datasets gives us a comprehensive history of individuals health profiles, care needs, history of health services and medication use, as well as aged care facility characteristics, but we are still limited to the content of these existing datasets, which do not have important more in-depth clinical content, psychosocial and well-being variables, or other potentially important predictors of the events studied. Finally, while a large number of Australians use residential respite care prior to entering permanent care, ${ }^{45}$ and therefore, had prior experience with RACFs, we focused on the transition to permanent care in our analysis. To determine whether prior care in an RACF influenced the risk of the outcomes studied we examined the incidence of unplanned hospitalisation and ED presentations in individuals with some respite care the year prior to entry and found it to be similar to the overall cohort $(17.3 \%$ vs $21.6 \%$, respectively).

Our study provides a population-based estimate of the incidence and predictive factors for unplanned hospitalisations and ED encounters in most individuals who entered an RACF in three Australians states covering 68\% of the Australians RACF residents over a 4-year period. Our findings are applicable to the rest of the Australian population and Western countries with similar aged care 
sectors with similarly ageing populations. Our models all appropriately address competing risk of mortality after RACF entry, which obviously affect the likelihood of the studied events. We have also used models that can moderately well categorise individuals according to their risk of unplanned hospitalisations and ED presentation and provide insightful information about the factors that contribute to these risks, which can be used by clinicians and aged care providers in their care planning and risk mitigation strategies.

\section{CONCLUSIONS AND IMPLICATIONS}

With over 70000 individuals entering RACF permanently every year in Australia alone and significant time dedicated to care needs assessment and planning, this is a useful time to characterise those at most risk of ED presentations and unplanned hospitalisations, which are commonplace in individuals entering care. Identifying those at highest risk can inform better monitoring and surveillance, preparation of providers, carers and clinicians regarding their care, and target some of the potentially modifiable factors associated with these events.

\section{Author affiliations}

${ }^{1}$ Allied Health and Human Performance, University of South Australia, Adelaide, South Australia, Australia

${ }^{2}$ Registry of Senior Australians, South Australian Health and Medical Research Institute, Adelaide, South Australia, Australia

${ }^{3}$ South Australian Health and Medical Research Institute, Adelaide, South Australia, Australia

${ }^{4}$ Southern Adelaide Local Health Network, SA Health, Adelaide, South Australia, Australia

${ }^{5}$ College of Medicine and Public Health, Flinders University, Adelaide, South Australia, Australia

${ }^{6}$ Northern Adelaide Local Health Network, SA Health, Adelaide, South Australia, Australia

${ }^{7}$ Central Adelaide Local Health Network, SA Health, Adelaide, South Australia, Australia

${ }^{8}$ College of Nursing and Health Sciences, Flinders University, Adelaide, South Australia, Australia

Correction notice This article has been corrected since it was first published. Twitter Maria C Inacio @mariacinacio and Janet K Sluggett @JanetSluggett

Acknowledgements We would like to acknowledge the Registry of Senior Australians' (ROSA) Steering Committee and the ROSA South Australian Health and Medical Research Institute Research Team for ensuring the success of the ROSA and support with this study. We also acknowledge the South Australian Government who provided us with support (2017-2021) through the Department for Innovation and Skills, and the Australian Institute of Health and Welfare (AlHW) for the linkage and construction of the input data, SA Health, NSW Ministry of Health, and VIC Department of Health and Human Services (DHHS) for the provision of the state-based data used in the ROSA with linkage via the AlHW, Centre for Health Record Linkage (CHeReL), the Centre for Victorian Data Linkage (CVDL) and SA NT DataLink.

Collaborators State Action on Avoidable Rehospitalisations and Unplanned Admission (STAAR-SA) Study Collaborators: Carmel McNamara, Clarabelle T Pham, $\mathrm{PhD}$, Jonathon Karnon, PhD, Elizabeth Lynch, PhD, Kate Laver, PhD, Jesmin Rupa, $\mathrm{PhD}$.

Contributors The following authors made substantial contributions to conception and design: $\mathrm{MCl}, \mathrm{RNJ}, \mathrm{GH}$ and MC. Acquisition of data: $\mathrm{MCl}$, SW and CW. Analysis and interpretation of data: MCl, RNJ, SW, JKS, JM, JF, AB, GH, MC, STAAR-SA Study Collaborators. The manuscript was drafted by $\mathrm{MCl}$ and RNJ and it was critically revised with input from SW, JKS, CW, JM, JF, AB, GH, MC, STAAR-SA
Study Collaborators. MCl is the study guarantor and accepts full responsibility for the finished work and/or the conduct of the study, had access to the data, and controlled the decision to publish.

Funding This project was supported by the Australian Government's Medical Research Future Fund (MRFF) as part of the Rapid Applied Research Translation program. This project is part of the work being undertaken by Health Translation SA. MCl is supported by The Hospital Research Foundation Mid-Career Fellowship (MCF-27-2019) and National Health and Medical Research Council (NHMRC) Investigator Grant (APP119378).

Disclaimer The funders had no role in the study design, methods, data analysis, decision publish or preparation of this manuscript.

Competing interests $\mathrm{CW}$ is a board member of the aged care organisation Helping Hand.

Patient consent for publication Not applicable.

Ethics approval This study has received ethics approval from the University of South Australia's Human Research Ethics Committee (Ref: 200487), Australian Institute of Health and Welfare Ethics Committee (Ref: E02018/1/418), South Australian Department for Health \& Wellbeing Human Research Ethics Committee (Ref: HREC/18/SAH/90) and New South Wales Population \& Health Services Research Ethics Committee (Ref: 2019/ETH12028).

Provenance and peer review Not commissioned; externally peer reviewed.

Data availability statement Data may be obtained from a third party and are not publicly available. The data for this study were obtained from the Australian Institute of Health and Welfare, Australian Government Department of Health and South Australia, Victoria, and New South Wales state health authorities and integrated by the Australian Institute of Health and Welfare, Centre for Health Record Linkage, and SA NT DataLink. These data were made available to the researchers under ethical, governance, and confidentiality agreements that do not allow public sharing.

Supplemental material This content has been supplied by the author(s). It has not been vetted by BMJ Publishing Group Limited (BMJ) and may not have been peer-reviewed. Any opinions or recommendations discussed are solely those of the author(s) and are not endorsed by BMJ. BMJ disclaims all liability and responsibility arising from any reliance placed on the content. Where the content includes any translated material, BMJ does not warrant the accuracy and reliability of the translations (including but not limited to local regulations, clinical guidelines, terminology, drug names and drug dosages), and is not responsible for any error and/or omissions arising from translation and adaptation or otherwise.

Open access This is an open access article distributed in accordance with the Creative Commons Attribution Non Commercial (CC BY-NC 4.0) license, which permits others to distribute, remix, adapt, build upon this work non-commercially, and license their derivative works on different terms, provided the original work is properly cited, appropriate credit is given, any changes made indicated, and the use is non-commercial. See: http://creativecommons.org/licenses/by-nc/4.0/.

ORCID iDs

Maria C Inacio http://orcid.org/0000-0001-8261-2665

Maria Crotty http://orcid.org/0000-0002-2996-5135

\section{REFERENCES}

1 Organisation for Economic Co-operation and Development. OECD STAT. long-term care resources and utilisation. long-term care recipients. Available: https://stats.oecd.org/Index.aspx?Queryld= 30142

2 Khadka J, Lang C, Ratcliffe J, et al. Trends in the utilisation of aged care services in Australia, 2008-2016. BMC Geriatr 2019;19:213.

3 Australian Government. Department of health. 2019-20 report on the operation of the aged care act, 1997. Available: https://www. gen-agedcaredata.gov.au/resources/reports-and-publications/2020/ november/2019\%E2\%80\%9320-report-on-the-operation-of-theaged-care-a

4 Australian Government. Australian Institute of Health and Welfare. Australia's health 2016. 2.1 How does Australia's health system work? Available: https://www.aihw.gov.au/getmedia/f2ae1191-bbf247b6-a9d4-1b2ca65553a1/ah16-2-1-how-does-australias-healthsystem-work.pdf.aspx

5 Inacio MC, Lang C, Bray SCE, et al. Health status and healthcare trends of individuals accessing Australian aged care programmes over a decade: the registry of senior Australians historical cohort. Intern Med J 2021;51:712-24. 
6 Hoffmann F, Allers K. Variations over time in the effects of age and sex on hospitalization rates before and after admission to a nursing home: a German cohort study. Maturitas 2017;102:50-5.

7 Boyd M, Broad JB, Zhang TX, et al. Hospitalisation of older people before and after long-term care entry in Auckland, New Zealand. Age Ageing 2016;45:558-63.

8 Hoffmann F, Allers K. Age and sex differences in hospitalisation of nursing home residents: a systematic review. BMJ Open 2016;6:e011912.

9 Hirdes JP, Heckman GA, Morinville A, et al. One way out? A multistate transition model of outcomes after nursing home admission. J Am Med Dir Assoc 2019;20:1425-31.

10 Welberry HJ, Jorm LR, Schaffer AL, et al. Psychotropic medicine prescribing and polypharmacy for people with dementia entering residential aged care: the influence of changing general practitioners. Med J Aust 2021;215:130-6.

11 Ferrah N, Ibrahim JE, Kipsaina C, et al. Death following recent admission into nursing home from community living: a systematic review into the transition process. J Aging Health 2018;30:584-604.

12 Haber SG, Wensky SG, McCall NT. Reducing inpatient hospital and emergency room utilization among nursing home residents. J Aging Health 2017:29:510-30.

13 Australian Institute of Health and Welfare. Admitted patient care 2017-18: Australian hospital statistics. health services series No. 90 Cat. no. HSE 225. AlHW. Available: https://www.aihw.gov.au/ getmedia/df0abd15-5dd8-4a56-94fa-c9ab68690e18/aihw-hse-225. pdf.aspx?inline=true

14 Commonwealth of Australia. Royal Commission into aged care quality and safety. research paper 18: hospitalisations in Australian Aged Care: 2014/15-2018/19. Available: https://agedcare. royalcommission.gov.au/sites/default/files/2021-02/research-paper18-hospitalisations-australian-aged-care.pdf

15 Brucksch A, Hoffmann F, Allers K. Age and sex differences in emergency department visits of nursing home residents: a systematic review. BMC Geriatr 2018;18:151

16 Australian Commission on Safety and Quality in Health Care. Safety issues at transitions of care. Consulation report on pain points relating to clinical information systems https://www.safetyandquality. gov.au/sites/default/files/migrated/Safety-issues-at-transitions-ofcare-consultation-report.pdf

17 Khadka J, Visvanathan $\mathrm{R}$, Theou $\mathrm{O}$, et al. Development and validation of a frailty index based on Australian aged care assessment program data. Med J Aust 2020;213:321-6.

18 Australian Bureau of Statistics. Census of population and housing: socio-economic indexes for areas (SEIFA), Australia, 2016. Available: https://www.abs.gov.au/AUSSTATS/abs@.nsf/Lookup/2033.0.55. 001Main+Features12016? OpenDocument

19 Hugo Centre for Migration and Population Research. Accessibility/ Remoteness index of Australia plus (ARIA+) 2016. The University of Adelaide. Available: https://www.adelaide.edu.au/hugo-centre/ services/aria

20 World Health Organization Collaborating Centre for Drug Statistics Methodology. ATC/DDD index, 2018. Available: https://www.whocc. no/atc_ddd_index/

21 Linjakumpu T, Hartikainen S, Klaukka T, et al. A model to classify the sedative load of drugs. Int J Geriatr Psychiatry 2003;18:542-4.

22 Hosmer D, Lemeshow S. Applied survival analysis: regression modeling of time to event data. New York, NY, USA: Wiley, 2008: 132-68.

23 Graverholt B, Riise T, Jamtvedt G, et al. Acute hospital admissions among nursing home residents: a population-based observational study. BMC Health Serv Res 2011;11:126.

24 O'Malley AJ, Caudry DJ, Grabowski DC. Predictors of nursing home residents' time to hospitalization. Health Serv Res 2011;46:82-104.

25 Ooi M, Lewis ET, Brisbane J, et al. Feasibility of using a risk assessment tool to predict Hospital transfers or death for older people in Australian residential aged care. A retrospective cohort study. Healthcare 2020;8 doi:10.3390/healthcare8030284
26 Seppala LJ, Wermelink AMAT, de Vries M, et al. Fall-Risk-Increasing drugs: a systematic review and meta-analysis: II. psychotropics. J Am Med Dir Assoc 2018;19:371.e11-371.e17.

27 Pharmaceutical Society of Australia. Medicine safety: aged care. Available: https://www.psa.org.au/wp-content/uploads/2020/02/ Medicine-Safety-Aged-Care-WEB-RES1.pdf

28 Australian Government, Department of Health. National aged care mandatory quality indicator program (Qi program). manual 2.0-Part A- final draft. Available: https://www.health.gov.au/sites/default/ files/documents/2021/04/national-aged-care-mandatory-qualityindicator-program-manual-national-aged-care-mandatory-qualityindicator-program-manual-2-0-part-a-final-draft.pdf [Accessed 30 Apr 2021].

29 Lalic S, Sluggett JK, Ilomäki J, et al. Polypharmacy and medication regimen complexity as risk factors for hospitalization among residents of long-term care facilities: a prospective cohort study. $J$ Am Med Dir Assoc 2016;17:1067.e1-1067.e6.

30 Green L, Selleck L, Gibbons M, et al. Does the evidence justify routine transfer of residents of aged care facilities for CT scan after minor head trauma? Intern Med J 2020;50:1048-52.

31 Sluggett JK, Lalic S, Hosking SM, et al. Root cause analysis of fall-related hospitalisations among residents of aged care services. Aging Clin Exp Res 2020;32:1947-57.

32 Maes ML, Fixen DR, Linnebur SA. Adverse effects of proton-pump inhibitor use in older adults: a review of the evidence. Ther Adv Drug Saf 2017;8:273-97.

33 Sluggett JK, Hendrix I, Bell JS. Evidence-based deprescribing of proton pump inhibitors in long-term care. Res Social Adm Pharm 2018;14:124-6.

34 Kim DH, Brown RT, Ding EL, et al. Dementia medications and risk of falls, syncope, and related adverse events: metaanalysis of randomized controlled trials. J Am Geriatr Soc 2011;59:10.1111/j.1532-5415.2011.03450.x:1019-31.

35 Hoffmann F, Strautmann A, Allers K. Hospitalization at the end of life among nursing home residents with dementia: a systematic review. BMC Palliat Care 2019;18:77.

36 Grabowski DC, Stewart KA, Broderick SM, et al. Predictors of nursing home hospitalization: a review of the literature. Med Care Res Rev 2008;65:3-39.

37 Tanuseputro P, Chalifoux M, Bennett C, et al. Hospitalization and mortality rates in long-term care facilities: does for-profit status matter? J Am Med Dir Assoc 2015;16:874-83.

38 Commonwealth of Australia. Royal Commission into aged care quality and safety. research paper 15: residential care quality indicator profile. Available: https://agedcare.royalcommission.gov. au/publications/research-paper-15-residential-care-quality-indicatorprofile

39 Inacio $\mathrm{M}$, Jorissen $\mathrm{RN}$, et al. The national spending and uptake of mobile radiology services in aged care facilities- an opportunity to improve access remains. Int Med J 2021.

40 Burkett EBD, Donovan R, Cranitch E, et al. Implementation tool-kit for residential aged care support services (RaSS). CARE-PACT and healthcare improvement unit, Queensland health. Available: https://clinicalexcellence.qld.gov.au/sites/default/files/2019-07/racfimplementation-toolkit.pdf

41 SA Ambulance Service. SA ambulance service fact sheet: extended care paramedics (ECPs)

42 Zhou H, Della PR, Roberts P, et al. Utility of models to predict 28-day or 30-day unplanned hospital readmissions: an updated systematic review. BMJ Open 2016;6:e011060.

43 Wallace E, Stuart E, Vaughan N, et al. Risk prediction models to predict emergency hospital admission in community-dwelling adults: a systematic review. Med Care 2014;52:751-65.

44 Inacio MC, Jorissen RN, Khadka J, et al. Predictors of short-term hospitalization and emergency department presentations in aged care. J Am Geriatr Soc 2021 doi:10.1111/jgs.17317

45 Harrison SL, Lang C, Whitehead C, et al. Residential Respite care use is associated with fewer overall days in residential aged care. $J$ Am Med Dir Assoc 2020;21:653-9. 